\title{
Reinforcement of Injectable Calcium Phosphate Cement by Gelatinized Starches
}

Huiling Liu ${ }^{1}$, Ying Guan ${ }^{2}$, Donglei Wei ${ }^{1}$, Chunxia Gao ${ }^{1}$, Huilin Yang ${ }^{1}$, Lei Yang ${ }^{1}$

${ }^{1}$ Institute of Orthopaedics, The First Affiliated Hospital, Soochow University, Suzhou, Jiangsu 215006, P.R. China

${ }^{2}$ College of Chemistry, Chemical Engineering and Material Science, Soochow University, Suzhou, Jiangsu 215123, P.R.

Statement of Purpose: Current injectable calcium phosphate bone cements (CPC) encounter the problems of low strength, high brittleness, and low cohesion in aqueous environment, which greatly hinder their clinical applications for loading-bearing bone substitution and minimally invasive orthopedic surgeries like percutaneous vertebroplasty or kyphoplasty. Because of this, many efforts have so far been made to increase CPC mechanical strengths and improve its cohesion and anti-collapsibility (also known as anti-washout property). In this study, a strategy of using food-grade, gelatinized starches to reinforce injectable CPC was proposed. The effects of various starch gels on CPC properties, including injectability, anti-collapsibility, compressive strength and modulus, strain energy density and cytotoxicity, were investigated.

Methods: Four types of starches, corn starch, crosslinked starch, cationic starch, and Ca-modified starch were studied. Corn starch, cross-linked starch, cationic starch were purchased and Ca-modified starch gel was prepared by treating the pristine waxy starch with calcium salts like $\mathrm{CaCl}_{2}$ and $\mathrm{Ca}\left(\mathrm{NO}_{3}\right)_{2}$ in the water at the temperature more than $60^{\circ} \mathrm{C}$. Formulation of CPC powder in this study contained $70 \mathrm{wt} . \% \beta$-tricalcium phosphate, $20 \mathrm{wt} . \%$ tetracalcium phosphate and $10 \mathrm{wt} . \%$ dicalcium phosphate dehydrate [1]. For preparing starchmodified CPC samples, dry CPC powder was mixed with different types of starch at two contents of $3 \mathrm{wt} . \%$ or 10 wt. $\%$. The mixture of dry powders was added to deionized water at liquid-to-solid ratios of 0.75 and 1.05 for the starch contents of $3 \mathrm{wt} . \%$ or $10 \mathrm{wt} . \%$, respectively. The starch suspension was then mechanically stirred and gelatinized at $80{ }^{\circ} \mathrm{C}$ to form a uniform paste. The $\mathrm{CPC} /$ starch pastes were cooled down to room temperature and then injected through a $1 \mathrm{~mL}$ syringe with an outlet diameter of $4.8 \mathrm{~mm}$, forming cylindrical paste bars for further testing.

Mechanical properties of the starch-modified CPCs were characterized by compression, soaking, and shaking tests. Hardened cement samples were cut into bars of 4.2 $\mathrm{mm}$ in diameter and $8.4 \mathrm{~mm}$ in length and then dried at 37 ${ }^{\circ} \mathrm{C}$ in air for 2 days. Compression tests were performed on a uniaxial mechanical tester operating at crosshead speed of $1 \mathrm{~mm} / \mathrm{min}$. For evaluating anti-collapsibility, the cement paste was injected into deionized water directly and soaked in the water for 7 days and then moved to an orbital shaker for shaking tests at the speed of $50 \mathrm{rpm}$ for 1 day and then at $100 \mathrm{rpm}$ for another day.

Microstructures of the starch-modified CPCs were evaluated by scanning electron microscopy (SEM). Cytotoxicity of the starch-modified CPC was evaluated by measuring proliferation of osteoblasts in the mixture of cement leaching solution and standard cell culture medium.
China

Results: All the four types of starches studied here resulted in the reinforcements of CPC to different extents, including increased compressive strengths and moduli (Figure 1). Specifically, adding $10 \mathrm{wt} . \%$ corn starch and 3 wt.\% Ca-modified starch leaded to the highest compressive strengths and moduli of the composite cements, with 6-fold increase in the compressive strength and at least 2-fold increase in moduli compared to pure $\mathrm{CPC}$, respectively. In addition, $\mathrm{CPC}$ modified with starch gels significantly improved the strain energy density of the cement.

Addition of starch gels with proper contents increased the injectability and anti-collapsibility of CPC.

Specifically, CPCs modified by corn, cationic and Camodified starches remained intact in water for up to 7 days and subsequently retained its integrity when further tested on an orbital shaker at speeds of $50 \mathrm{rpm}$ and then $100 \mathrm{rpm}$ each for a day.

Osteoblast proliferation tests on the leaching solution of the cements showed that starch gels had no adverse effects on osteoblast proliferation up to 3 days and all starch-modified CPC revealed similar cytocompatibility as pure CPC. Cell proliferation rates on the CPC incorporated with cross-linked, cationic and Ca-modified starches were even higher than that on the phosphate buffered solution (PBS) control.

Two possible mechanisms, reinforcement by the second phase of starch and strong interlocking of apatite crystals, were proposed based on the results of starch gel zeta potential, viscosity, cement microstructures from SEM observations, and resultant mechanical properties.

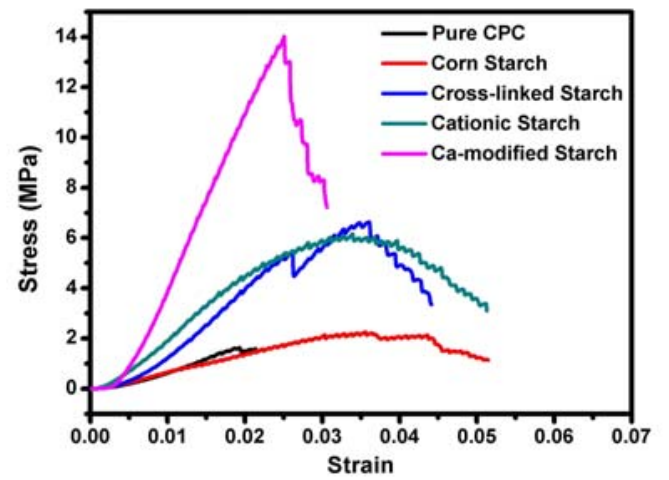

Figure 1. Typical compressive stress-strain curves of CPC reinforced by 3 wt. \% starches.

Conclusions: Incorporating gelatinized starches was an effective, facile and bio-friendly strategy to reinforce injectable CPC and improve its mechanical stability, and thus, should be further studied and developed.

\section{References:}

[1] Zhang J, Liu W, Schnitzler V, Tancret F, Bouler JM. Acta Biomater 2014; 10 (3): 1035-1049. 